\title{
RR Lyrae binary systems in the Galactic bulge
}

\author{
Gergely Hajdu ${ }^{1,2, \star}$ and Márcio Catelan ${ }^{1,2, \star \star}$ \\ ${ }^{1}$ Pontificia Universidad Católica de Chile, Facultad de Física, Instituto de Astrofísica, Av. Vicuña Mackenna \\ 4860, 782-0436 Macul, Santiago, Chile \\ ${ }^{2}$ Millennium Institute of Astrophysics, Santiago, Chile
}

\begin{abstract}
.
The possibility of a direct mass measurement of RR Lyrae variable stars in binary systems has remained elusive for many years, due to the apparent paucity of such systems. Motivated by our recent success of finding a population of high confidence candidates, we have been continuing the search for RR Lyrae variables in binary systems towards the Galactic bulge. We describe the preliminary results of our project in these proceedings.
\end{abstract}

\section{Introduction}

Direct mass measurements of variable stars provide an indispensable tool to test the models of stellar pulsation and evolution. Previously only one RR Lyrae star was known to be in a binary system with high confidence $([5,10])$. This has changed when we announced 20 candidate binary systems, using OGLE observations of RR Lyrae stars towards the bulge ([1]). Furthermore, an independent analysis of field RR Lyrae has also found a few plausible binary systems ([6]). Finding new binary RR Lyrae is paramount to understand their paucity, as well as to select suitable systems for follow-up observations.

\section{Analysis}

We are continuing our search for variations caused by the light-time effect ([2]) in the O-C diagrams ([9]) of the combined $I$ band light curves of RR Lyrae stars (RRab subtype) of the OGLE-II, III and IV projects $([7,8])$ towards the Galactic bulge, utilizing the same method as described in [1]. Compared to the limited sample of the previous study (1952 stars), we have analyzed more objects (5948), by relaxing the quality requirements of the analyzed light curves.

During our analysis, we have inspected the generated $\mathrm{O}-\mathrm{C}$ diagrams and folded light curves of the stars one by one. In many cases, the $\mathrm{O}-\mathrm{C}$ diagrams show periodic signals similar to those caused by the light-time effect, but inspection of the folded light curve revealed the presence of the Blazhko effect ([4]), usually with the same, long-term period as the $\mathrm{O}-\mathrm{C}$ oscillations. In some cases the amplitude modulation caused by the Blazhko effect is on the level of $\sim 0.01 \mathrm{mag}$, despite the thousand-day long periods. Based on this, we warn against the overinterpretation of $\mathrm{O}-\mathrm{C}$ diagrams derived from timing of the maxima of RR Lyrae stars alone. Unfortunately, this could be a plausible explanation for some of the bright RR Lyrae binary candidates published by [6].

$\star$ ghajdu@astro.puc.cl

$\star \star$ mcatelan@astro.puc.cl 


\section{Binary candidates}

We have found $\sim 35$ new good binary candidates in the analyzed sample, after discarding all variables showing the Blazhko effect. Preliminary analysis indicates the following results:

- The distribution of orbital periods is similar to that in our original study ([1]); the bulk of the candidates is in the $8-12 \mathrm{yr}$ range. Binaries with longer periods remain undetectable with the light-time effect method due to the limited time coverage of the observations.

- There are no short-period ( $<$ few yr) RR Lyrae binaries; this is probably a side effect of binary co-evolution prohibiting the formation of normal RR Lyrae variables in binaries with short orbital periods.

- The frequency of RR Lyrae binaries is compatible with a lower limit of $4 \%$ as in [1], when taking the lower average quality of the data into consideration.

- The majority of the candidates have low minimum companion masses, making radial velocity follow-up observations challenging; the expected radial velocity signal is only a few $\mathrm{km} \mathrm{s}^{-1}$, which is superposed on the signal of the pulsation with tens of $\mathrm{km} \mathrm{s}^{-1}$.

We are organizing follow-up observations of our best binary candidates. An interesting possibility of binary stellar evolution is that for a subset of these binaries the secondary body in the system could be the degenerate remnant of an originally higher-mass star. If the star went through the asymptotic giant branch (AGB) phase, it could have polluted the photospehere of the RR Lyrae with $s$-process elements, producing a so-called CEMP (Carbon-Enhanced Metal-Poor) star. Up to this date, several RR Lyrae variables have been found with such elemental enhancements ([3]); however, none of them has been shown to still reside in a physical binary with the white dwarf remnant of the AGB star. The detection of carbon enhancement by observing the $\mathrm{CN}$ band using low-resolution spectroscopy can easily prove the existence of degenerate companions for our binary candidates, at a moderate expense of telescope time on $8 \mathrm{~m}$-class telescopes. In turn, these stars would be the very first RR Lyrae variables spectroscopically confirmed to reside in a binary system.

Acknowledgments: Support for this project is provided by the Ministry for the Economy, Development, and Tourism's Programa Iniciativa Milenio through grant IC 120009; by Proyecto Basal PFB-06/2007; by Proyectos Fondecyt \#1141141 and \#1171273; and by CONICYT-PCHA/Doctorado Nacional grant \#2014-63140099.

\section{References}

[1] Hajdu, G., Catelan, M., Jurcsik, J., Dékány, I., Drake, A. J., \& Marquette, J.-B., MNRAS, 449, L113 (2015)

[2] Irwin, J. B., ApJ, 116, 211 (1952)

[3] Kennedy, C. R., Stancliffe, R. J., Kuehn, C., et al., ApJ, 787, 6 (2014)

[4] Kovács, G., in Stellar Pulsation: Challanges for Theory and Observation, ed. J. A. Guzik \& P. A. Bradley, AIP Conference Proceedings, 1170, 261 (2010)

[5] Liška, J., Mikulášek, Z., Zejda, M., \& Chrastina, M., A\&A, 589, 94 (2016)

[6] Liška, J., Skarka, M., Zejda, M., Mikulášek, Z., \& de Villiers, S. N., MNRAS, 459, 4360 (2016)

[7] Soszyński, I., Dziembowski, W. A., Udalski, A., et al., AcA, 61, 1 (2011)

[8] Soszyński, I., Udalski, A., Szymański, M. K., et al., AcA, 64, 177 (2014)

[9] Sterken C., in The Light-Time Effect in Astrophysics, ed. C. Sterken, ASP Conference Series, 335, 3 (2005)

[10] Wade, R. A., Donley, J., Fried, R., White, R. E., \& Saha, A., AJ, 118, 2442 (1999) 